\title{
Real-Time Disturbance Detection Using STFT Method in Microgrids
}

\author{
${ }^{1}$ Alper Yilmaz and *2Gökay Bayrak \\ ${ }^{1}$ Bursa Technical University, Department of Electrical and Electronics Engineering, Turkey \\ *2 Bursa Technical University, Department of Electrical and Electronics Engineering, Turkey
}

\begin{abstract}
Power quality disturbances are the main concerns to be eliminated in microgrids and decrease the power quality and reliability of the grid. Numerous methods based on signal processing have been proposed in the literature for the detection of power quality disturbances. In this study, the proposed STFT-based method is applied to the voltage signal in real-time at the point of PCC in microgrids. By using the proposed method, it is tried to detection the sudden frequency changes and the over/under voltage events in case of fault conditions. As a result, the proposed method can detect faults in microgrids a very short time and with high accuracy within the limit values specified in international standards.
\end{abstract}

Keywords: Fault Detection, Short Time Fourier Transform, Microgrids, Real-time

\section{Introduction}

Microgrids, which are becoming more popular in the world, play an important role in overcoming the global energy crisis [1]. It is important to detect power quality disturbances quickly and reliably to ensure reliability and continuity of the power system [9]. Faults are the biggest obstacle to providing uninterrupted energy to the user in an electrical grid. Fault detection should be done as soon as possible in the most accurate way, and the power system should be affected with the least possible damage. In recent years, although there has been an increase in studies on faults and power quality issues in microgrids, there are a limited number of real-time signal processing-based studies that detect faults automatically. Most of the studies have not been able to go beyond simulation and focused on detecting a limited number of fault types.

Numerous methods based on signal processing have been proposed in the literature for the detection of power quality disturbances [10,11]. In [2], the voltage sag event was detected using a short-time Fourier transform (STFT). The test results show that voltage sag events are detected with high accuracy independent of harmonic and noise. In another study [3], faults in HVDC systems were detected using STFT. Tests were performed in PSCAD and MATLAB simulation environments. It is seen that STFT using the frequency parameter, can effectively detect the DC fault very fast $(<; 1.5 \mathrm{~ms})$. The STFT method has also been used to characterize serial arc faults [4]. In this study, the STFT algorithm used the Hanning window function and its $25 \mathrm{~Hz}$ frequency-resolution. The results from the test data indicate that event detection is performed with high accuracy.

*Corresponding author: Address: Department of Electrical and Electronics Engineering Bursa Technical University, Bursa /TURKEY. E-mail address: gokay.bayrak@btu.edu.tr 
In the [5] in which an algorithm was proposed for the classification of power quality disturbances, histogram graphs of the properties were obtained using STFT. Afterward, classification is performed using neural networks. In the simulation study [6], an STFT-based algorithm for the classification of faults in transmission lines is proposed. In the study of transient event analysis in DC systems [7], the proposed STFT-based algorithm can detect low-impedance faults within $1 \mathrm{~ms}$ and high-impedance faults in $2 \mathrm{~ms}$ in this study. The most commonly used methods for the detection of power quality events, STFT and wavelet filters, were tested on a 13bar power system in [8]. Results show the advantages and drawbacks of each signal processing technique.

In this study, the proposed STFT-based method is applied to the voltage signal at the point of common coupling (PCC) received in real-time via electronic measurement card and data acquisition card for different fault conditions. By using the proposed method, it is tried to detection the sudden frequency changes and the over/under voltage events in case of fault conditions. The proposed method was also experimentally verified with software developed with Labview. As a result, the proposed method can detect faults in microgrids a very short time and with high accuracy within the limit values specified in international standards.

\section{Short-Time Fourier Transform}

The Fourier transform (FT) method, which is the most widely used signal processing technique which was founded by Joseph Fourier, decomposes the signal into sine waveforms at different frequencies and provides a relationship between the time-frequency regions of the signal. That is, the time-based signal in the frequency domain corresponds to the FT. The FT formula is specified in Eq. (2.1).

$$
F(\omega)=\int_{-\infty}^{\infty} f(t) e^{-j \omega t} d t, \quad \omega \in(-\infty, \infty)
$$

FT results, which provide a general approach to signal signaling, do not allow for the local examination of frequency components. Therefore, FT cannot be used in short-term voltage changes and transient events because the signal is variable over time and contains sudden changes. The problem of not being able to examine the signals with variable properties by the FT method was solved by the windowing method, which allows the signal to be divided into smaller components. In this transformation called the STFT, the signal is decomposed into short term signals by windowing, and FT is calculated for each window. STFT is calculated in Eq. (2.2).

$$
F(\omega)=\int_{-\infty}^{\infty} f(t) \omega(t-\tau) e^{-j \omega t} d t, \quad \omega \in(-\infty, \infty)
$$

Here the signal $f(t)$ maps the time-frequency plane by multiplying the sliding window $(t-\tau)$ function selected by the user for analysis. The window functions commonly used in the analysis 
of power quality events are Bartlett, Hanning, Kaiser, rectangular, and triangular window functions. The STFT establishes a full relationship between time and frequency and allows the frequency at which time the signal is generated, depending on the size of the windowing. The window size used is fixed, and the frequency resolution of the signal increases as the window size increases, while the time resolution increases as the window size decreases.

\section{Proposed STFT-based Fault Detection Method}

STFT, a time-frequency-based analysis, establishes a complete relationship between time and frequency, allowing the frequency at which time the signal occurs depending on the windowing dimension. For this purpose, the interface of the Labview program developed for real-time STFT signal analysis is given in Figure 1.

In the STFT analysis of the voltage signal received via the DAQ card, the signal sampling frequency, the window function, and the width to be used for the STFT analysis can be set via the interface. The voltage waveform and frequency-power spectrum graph can also be accessed via the interface designed for STFT analysis.

In the analysis of the power quality event signals to be examined in this section, the Hanning function was selected as the windowing function, and two different windowing lengths $\mathrm{L}=32$ and $\mathrm{L}=64$, were performed to examine the effect of window length.

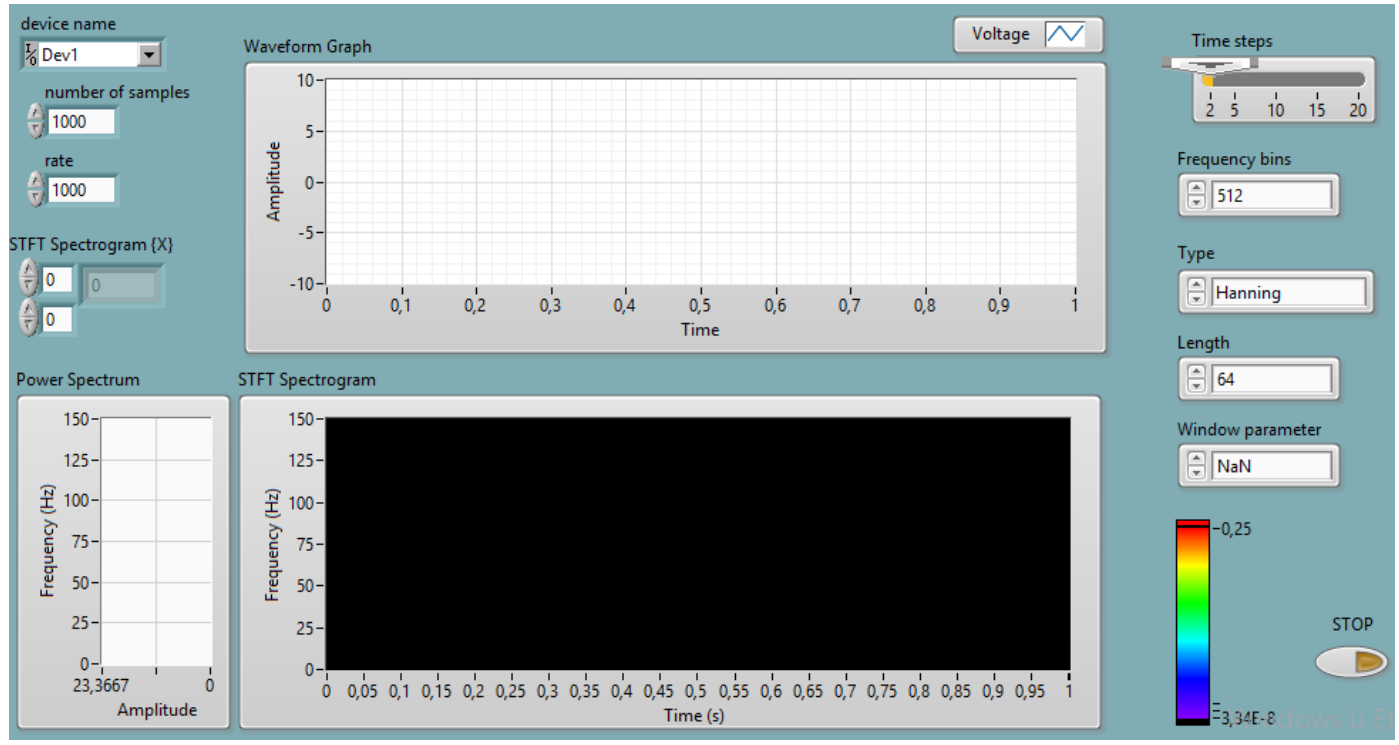

Figure 1. The interface of the Labview program

\section{Results}

In this part of the study, the proposed STFT-based detection method for different fault conditions has been tested. First, the scalogram of the signal and the power spectrum are plotted under nominal conditions. Afterward, tests were performed with different scenarios for sudden frequency changes and over/under voltage conditions. 
Case 1: For the analysis of the nominal voltage signal with STFT, the Hanning function was selected as the windowing function, and analysis was performed for two different windowing widths, $\mathrm{L}=32$, and $\mathrm{L}=64$. Figure 2 shows the STFT spectrogram graphs performed after the analysis. STFT analysis of the nominal voltage signal has shown in Figure 2.
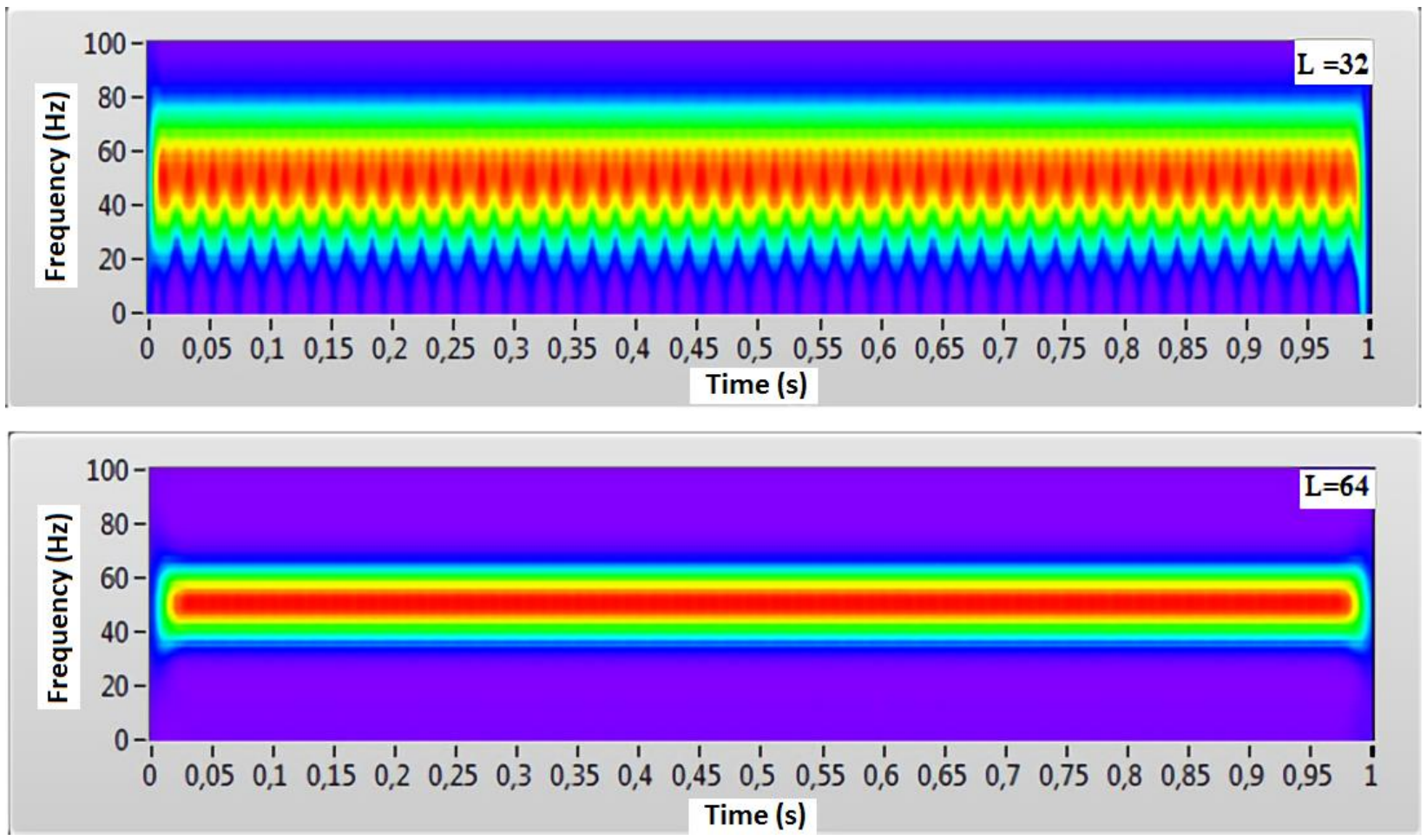

Figure 2. STFT analysis of nominal voltage signal

Case 2: Sudden frequency change is the deviation of the frequency from the nominal value in the distributed power system. Depending on the frequency change, the transition time of the microgrid system to island mode changed. For the detection of over/under frequency change by STFT analysis, Hanning function was selected as the windowing function, and analysis was performed for two different windowing lengths, $\mathrm{L}=32$ and $\mathrm{L}=64$. For analysis, the grid frequency is $50 \mathrm{~Hz}$, and the sampling frequency is $1 \mathrm{kHz}$. In Figure 3, a sudden frequency drop occurred in the voltage signal received on the card at $500 \mathrm{~ms}$, and as a result, the under frequency limit value was exceeded. With this decrease in frequency value, the new frequency value was $47.5 \mathrm{~Hz}$.

Case 3: The over and under-voltage fault is the deviation of the voltage from the nominal value in the distributed power system, resulting from overloaded motors, overloading, and faults along the line. The STFT spectrogram graph after the analysis performed for the overvoltage fault is given in Figure 4. The voltage amplitude of the grid frequency is $50 \mathrm{~Hz}$, and after 350th ms, the voltage amplitude increases to $120 \%$ of the nominal value. Results of STFT analysis for different windowing lengths are given in Figure 4. 

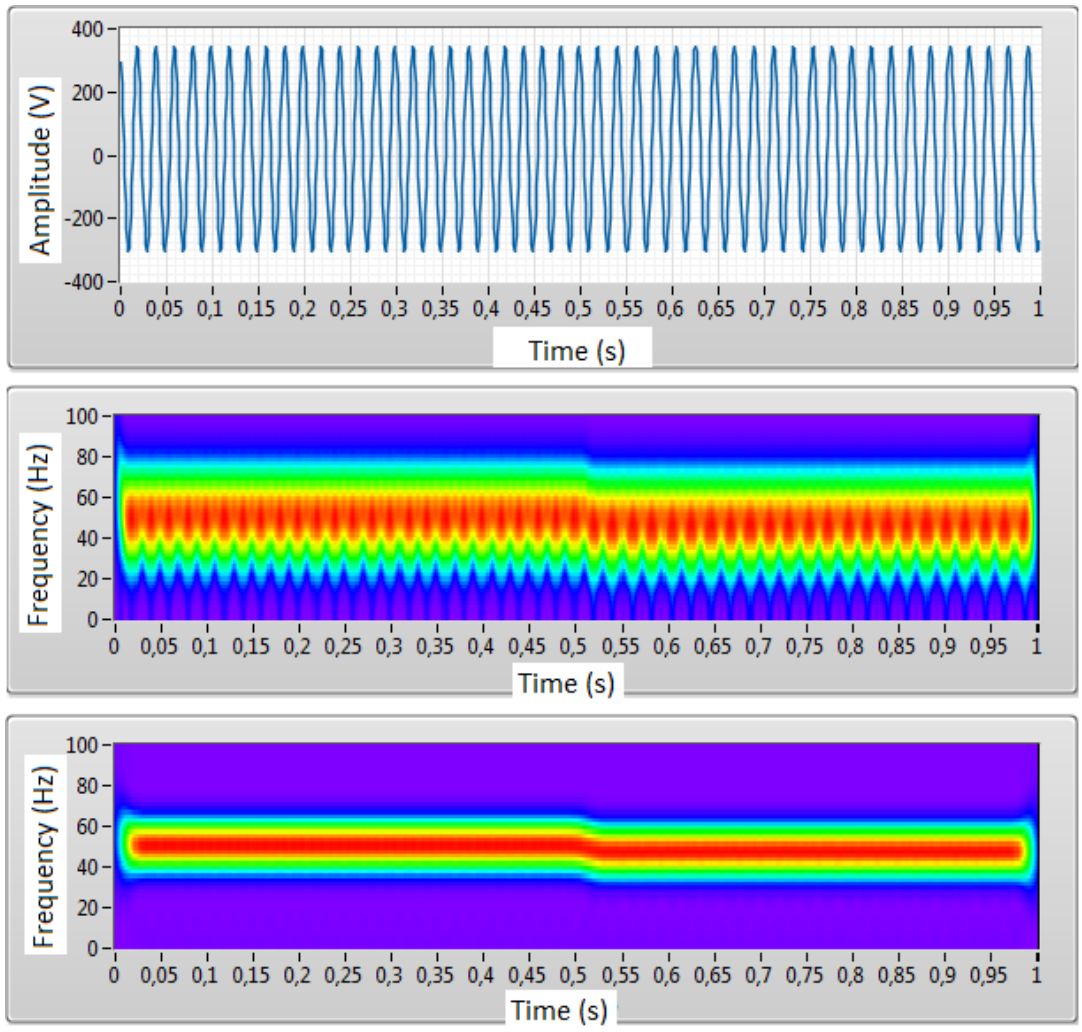

Figure 3. STFT analysis of under frequency fault
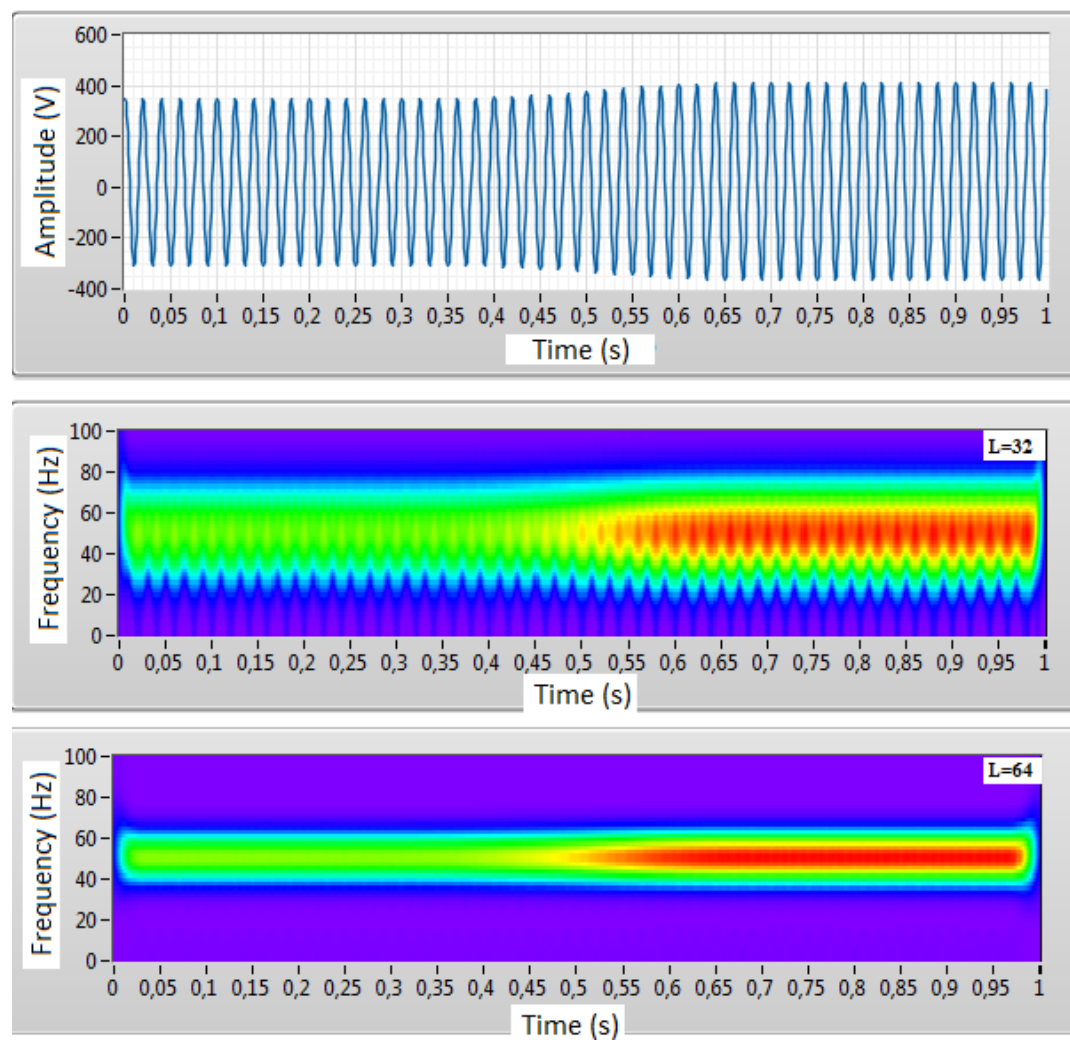

Figure 4. STFT analysis of overvoltage fault 
Case 4: STFT spectrogram graph formed after the analysis performed for the under-voltage event is given in Figure 5. After 375th ms, the voltage amplitude value decreases to $75 \%$ of the nominal value. At this point, the fault of the under-voltage fault is detected properly by the proposed real-time STFT-based detection method.
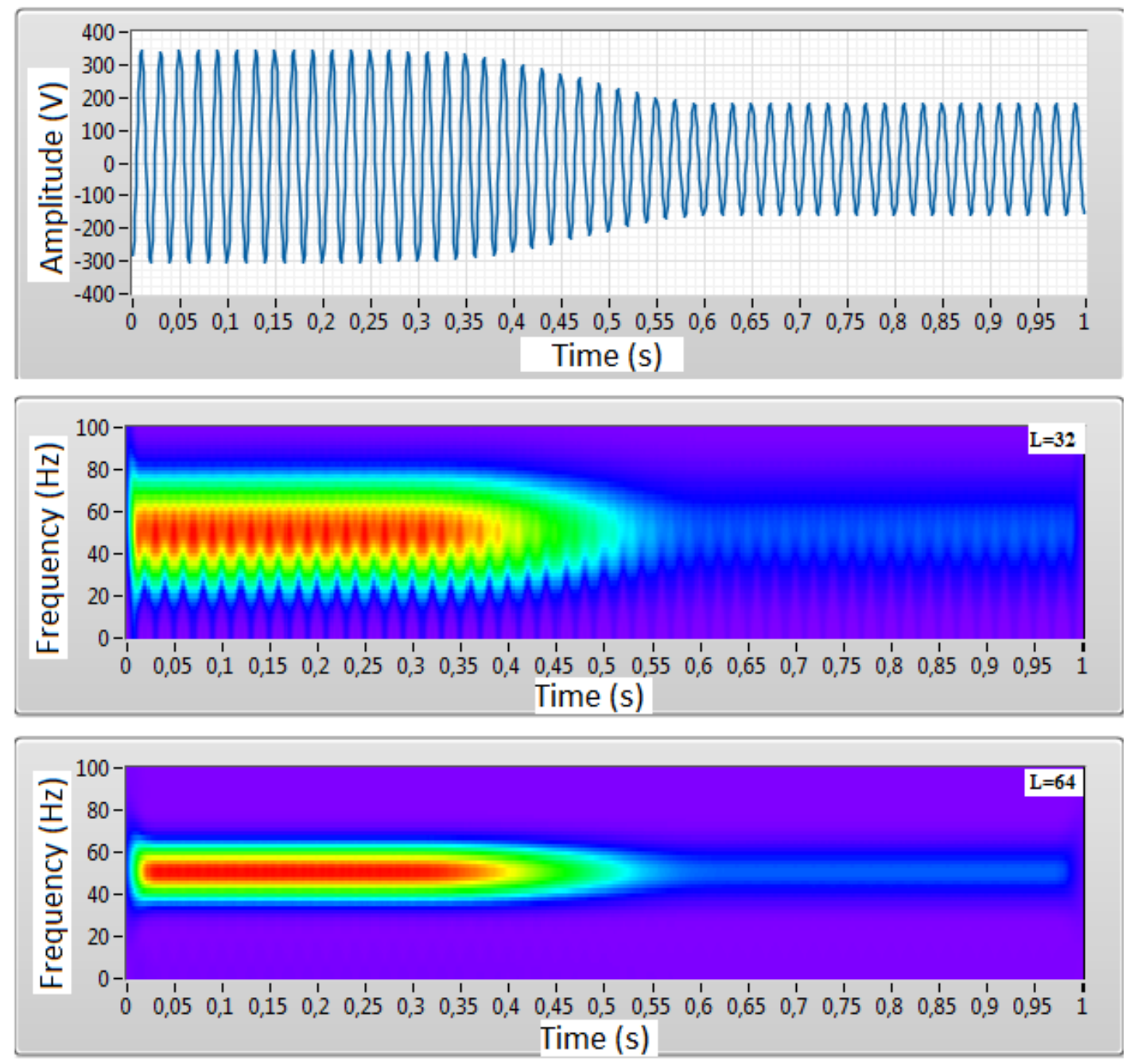

Figure 5. STFT analysis of under-voltage fault

\section{Discussion and Conclusion}

In recent years, although there has been an increase in studies on faults and power quality issues in microgrids, there are a limited number of real-time signal processing-based studies that detect faults automatically. In this study, the proposed STFT-based method is applied to the voltage signal in real-time at the point of PCC in microgrids. By using the proposed method, it is tried to detection the sudden frequency changes and the over/under voltage events in case of fault conditions. As a result, the proposed method can detect faults in microgrids a very short time and with high accuracy within the limit values specified in international standards. 


\section{References}

[1] Hamzaoui, I., Bouchafaa, F., \& Hadjammar, A. (2011). Investigation of the behavior of a three-phase grid-connected photovoltaic system to control active and reactive power with DPC, Energy Procedia, 6, 493-502.

[2] Fengzhan, Z., \& Rengang, Y. (2007). Voltage sag disturbance detection based on shorttime Fourier transform. Proceedings of the CSEE, 27(10), 28-34.

[3] Yeap, Y. M. \& Ukil, A. (2016, July). Fault detection in the HVDC system using a shorttime Fourier transform. In 2016 IEEE Power and Energy Society General Meeting (PESGM) (pp. 1-5). IEEE.

[4] Hong, C., Xiaojuan, C., Wei, X., \& Cong, W. (2009, December). Short-time Fourier transform-based analysis to characterization of series arc fault. In 2009 2nd International Conference on Power Electronics and Intelligent Transportation System (PEITS) (Vol. 1, pp. 185-188). IEEE.

[5] Valtierra-Rodriguez, M., de Jesus Romero-Troncoso, R., Osornio-Rios, R. A., \& GarciaPerez, A. (2013). Detection and classification of single and combined power quality disturbances using neural networks. IEEE Transactions on Industrial Electronics, 61(5), 2473-2482.

[6] Han, J., Kim, W. K., Lee, J. W., \& Kim, C. H. (2012). Fault type classification in transmission line using STFT.

[7] Satpathi, K., Yeap, Y. M., Ukil, A., \& Geddada, N. (2017). Short-time Fourier transformbased transient analysis of VSC interfaced point-to-point dc system. IEEE Transactions on Industrial Electronics, 65(5), 4080-4091.

[8] Jurado, F., \& Saenz, J. R. (2002). Comparison between discrete STFT and wavelets for the analysis of power quality events. Electric Power Systems Research, 62(3), 183-190

[9] Bayrak, G., \& Yilmaz, A. (2019). Assesment of Power Quality Disturbances For Grid Integration of PV Power Plants. Sakarya University Journal of Science, 23(1), 1-1.

[10] Bayrak, G. (2018). Wavelet transform-based fault detection method for hydrogen energybased distributed generators. International Journal of Hydrogen Energy, 43(44), 20293 20308.

[11] Yilmaz, A., \& Bayrak, G. (2019). A real-time UWT-based intelligent fault detection method for PV-based microgrids. Electric Power Systems Research, 177, 105984. 